\title{
Periodontal Screening and Recording Code 4
}

National Cancer Institute

\section{Source}

National Cancer Institute. Periodontal Screening and Recording Code 4. NCI Thesaurus.

Code C114652.

Indicates that the deepest probing depth in the sextant is greater than $5.5 \mathrm{~mm}$. 